\title{
Searches for additional charged Higgs bosons in the MSSM
}

\author{
Lluïsa-Maria Mir on behalf of the ATLAS Collaboration* \\ Institut de Física d'Altes Energies \\ E-mail: mir@ifae.es
}

\begin{abstract}
Searches for a charged Higgs boson in various decay channels using $36.1 \mathrm{fb}^{-1}$ of $p p$ collision data at a centre-of-mass energy of $13 \mathrm{TeV}$ collected with the ATLAS detector at the Large Hadron Collider are presented. No significant excess above the background-only hypothesis is observed and upper limits on the production cross-section times the branching ratio of the charged Higgs boson as a function of its mass are derived. The results are interpreted in benchmark scenarios of the Minimal Supersymmetric Standard Model.
\end{abstract}

The 39th International Conference on High Energy Physics (ICHEP2018)

4-11 July 2018

Seoul, Korea

${ }^{*}$ Speaker. 
No charged fundamental scalar bosons exist in the Standard Model (SM), but many theories beyond the SM include an extended Higgs sector with at least one pair of charged Higgs bosons. In CP-conserving two-Higgs-doublet models (2HDM) the properties of the charged Higgs boson ${ }^{1}$ depend on its mass, the mixing angle of the neutral CP-even Higgs bosons, $\alpha$, and the ratio of the vacuum expectation values of the two Higgs doublets, $\tan \beta$. In these proceedings, a search for $H^{+}$production in $p p$ collisions at a centre-of-mass-energy of $13 \mathrm{TeV}$ collected with the ATLAS detector [1] at the Large Hadron Collider (LHC) is presented. The analysis uses $36.1 \mathrm{fb}^{-1}$ of $p p$ data recorded during 2015 and 2016, and targets the $H^{+} \rightarrow \tau \nu$ [2] and $H^{+} \rightarrow t b$ [3] decays.

Depending on the assumption made for the decay mode of the $W$ boson originating from the top-quark produced together with the $H^{+}$, two final states are studied in the $H^{+} \rightarrow \tau v$ search: $\tau+$ jets if the $W$ boson decays into a quark pair, or $\tau+$ lepton if the $W$ boson decays into an electron or muon and neutrino(s). Only $\tau$-leptons decaying to hadrons are considered. Events with $\tau+$ jets are recorded using a missing transverse energy $\left(E_{\mathrm{T}}^{\text {miss }}\right)$ trigger with varying threshold depending on the data-taking period, and only events with at least one $\tau$ candidate with transverse momentum $\left(p_{\mathrm{T}}\right)$ larger than $40 \mathrm{GeV}$ are accepted. The events must contain at least three jets with $p_{\mathrm{T}}$ larger than $25 \mathrm{GeV}$ of which at least one has originated from a $b$-quark, have $E_{\mathrm{T}}^{\text {miss }}$ larger than $150 \mathrm{GeV}$, and transverse mass of the highest- $p_{\mathrm{T}} \tau$ candidate and $E_{\mathrm{T}}^{\text {miss }}$ larger than $50 \mathrm{GeV}$. No electrons or muons with $p_{\mathrm{T}}$ larger than $20 \mathrm{GeV}$ are allowed. $\tau+$ lepton events are recorded using single-lepton triggers and only events with exactly one electron or muon with $p_{\mathrm{T}}$ larger than $30 \mathrm{GeV}$ matched to the trigger object are considered. Exactly one $\tau$-lepton candidate with $p_{\mathrm{T}}$ larger than $30 \mathrm{GeV}$ and electric charge opposite to that of the lepton, and at least one jet with $p_{\mathrm{T}}$ larger than $25 \mathrm{GeV}$ and identified as originating from a $b$-quark must be present in the event. Finally, the $E_{\mathrm{T}}^{\text {miss }}$ must exceed $50 \mathrm{GeV}$. Backgrounds with prompt taus, like those in $W \rightarrow \tau \nu$ and $t \bar{t}$ events, are modelled with simulation, and the normalisation of the latter is corrected with data. Backgrounds with electrons misidentified as taus are estimated with simulation and validated in a $Z \rightarrow e^{+} e^{-}$control region (CR). Backgrounds where jets are misidentified as taus are estimated from data using a data-driven fake-factor method [2].

The search is performed in the $90-2000 \mathrm{GeV}$ mass region, including for the first time the mass region around the top-quark mass [4]. Multivariate techniques are used to separate signal from background and the parameters of interest, e.g. $\sigma\left(p p \rightarrow t b H^{+}\right) \times \mathscr{B}\left(H^{+} \rightarrow \tau v\right)$, are obtained from a simultaneous fit together with several nuisance parameters that encode statistical and systematic uncertainties. The compatibility of the data with background-only and signal+background hypotheses is computed from a profile likelihood ratio. Three signal regions (SR), $\tau+$ jets, $\tau+$ electron and $\tau+$ muon, and one CR enriched in $t \bar{t}$ events are considered simultaneously in the fit. The fitted distributions are binned boosted decision tree (BDT) scores in the SR and the number of events in the CR.

Data are found to be consistent with the background-only hypothesis and exclusions limits are set at the $95 \%$ confidence level (CL) by using the $\mathrm{CL}_{\mathrm{S}}$ procedure [5] on $\sigma\left(p p \rightarrow t b H^{+}\right) \times \mathscr{B}\left(H^{+} \rightarrow\right.$ $\tau \nu)$ for the full mass range and on $\mathscr{B}\left(t \rightarrow b H^{+}\right) \times \mathscr{B}\left(H^{+} \rightarrow \tau \nu\right)$ in the low $H^{+}$mass range. Figure 1 shows the expected and observed exclusion limits as a function of the $H^{+}$mass hypothesis.

\footnotetext{
${ }^{1}$ For simplicity in the following charged Higgs bosons are denoted $H^{+}$, with the charge-conjugate $H^{-}$always implied.
} 
The observed limit on the $\sigma\left(p p \rightarrow t b H^{+}\right) \times \mathscr{B}\left(H^{+} \rightarrow \tau v\right)$ ranges from $4.2 \mathrm{pb}$ to $2.5 \mathrm{fb}$ at $90 \mathrm{GeV}$ and $2000 \mathrm{GeV}$, respectively, and the limit on $\mathscr{B}\left(t \rightarrow b H^{+}\right) \times \mathscr{B}\left(H^{+} \rightarrow \tau v\right)$ from $0.25 \%$ to $0.031 \%$ at $90 \mathrm{GeV}$ and $160 \mathrm{GeV}$, respectively.
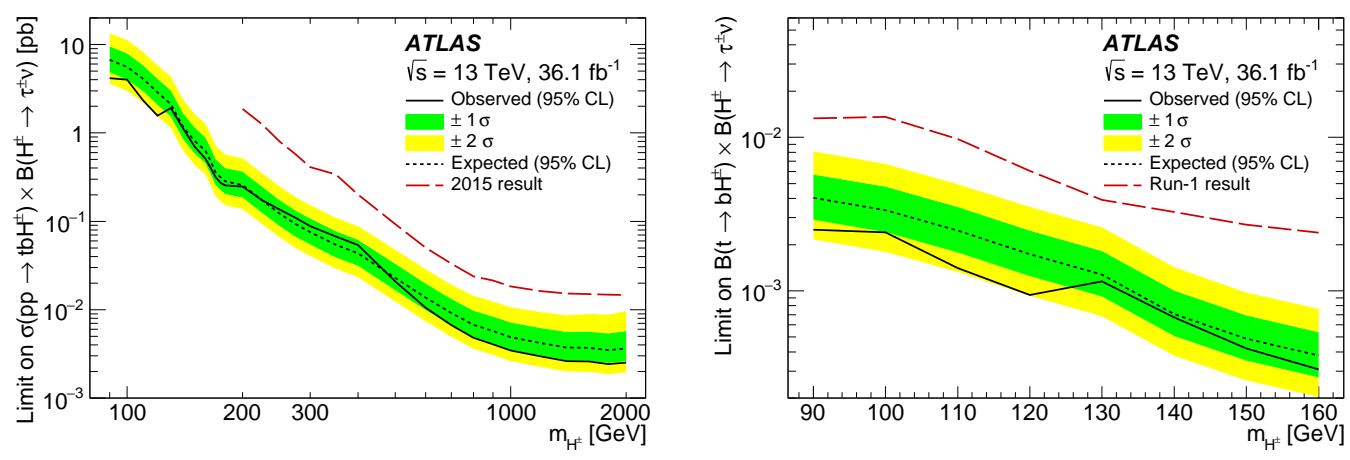

Figure 1: Observed and expected 95\% $\mathrm{CL}$ exclusion limits on $\sigma\left(p p \rightarrow t b H^{+}\right) \times \mathscr{B}\left(H^{+} \rightarrow \tau v\right)$ (left) and $\mathscr{B}\left(t \rightarrow b H^{+}\right) \times \mathscr{B}\left(H^{+} \rightarrow \tau v\right)$ (right) as a function of the $H^{+}$boson mass [2].

Charged Higgs decaying to a top-quark and a bottom-quark are recorded with single-lepton triggers. Events are required to have one or two electrons or muons, with the leading lepton matched to the lepton trigger and a $p_{\mathrm{T}}$ larger than $27 \mathrm{GeV}$. The second lepton must have opposite charge and $p_{\mathrm{T}}$ larger than $10 \mathrm{GeV}(15 \mathrm{GeV}$ in events with two electrons). At least three (five) jets are required in the events with two (one) leptons, of which at least two (three) must have originated from a $b$-quark. In the $e e$ and $\mu \mu$ channels the dilepton invariant mass must be incompatible with the $J / \psi$ and $Z$ masses. The selected events are classified into SR and CR according to the multiplicity of the jets and that of jets originated from a $b$-quark. The signal fraction is very small even in the most sensitive regions, and $t \bar{t}+$ jets events constitute the largest background. To improve its modelling, it is subdivided into $t \bar{t}+$ light, $t \bar{t} \geq 1 c$ and $t \bar{t} \geq 1 b$ categories, and the latter is reweighted to an NLO prediction of $t \bar{t} b \bar{b}$ including parton shower and hadronisation. A second type of reweighting is performed on several kinematical variables sequentially. Finally, since the number of events with high leading jet $p_{\mathrm{T}}$ is overestimated in the simulation, a reweighting function for the leading jet $p_{\mathrm{T}}$ is derived by comparing simulation and data [3]. Multi-jet events are modelled with data using a matrix method in events with one lepton and with simulation in events with two leptons. A different discriminant BDT is defined for each $\mathrm{H}^{+}$mass hypothesis and SR, which are used, together with the overall normalisation in the $\mathrm{CR}$, in a profile likelihood as in the $H^{+} \rightarrow \tau v$ analysis. For mass hypotheses up to $300 \mathrm{GeV}$, the BDTs for events with one lepton include a kinematic discriminant that reflects the compatibility of a given event with the $H^{+}$and $t \bar{t}$ hypotheses. The search is performed in the $200-2000 \mathrm{GeV}$ mass region. No significant excess above the bakground-only hypothesis is observed and exclusion limits are derived for $\sigma\left(p p \rightarrow t b H^{+}\right) \times \mathscr{B}\left(H^{+} \rightarrow t b\right)$ as a function of the $H^{+}$mass, which range from $2.9 \mathrm{pb}$ at $200 \mathrm{GeV}$ to $0.070 \mathrm{pb}$ at $2000 \mathrm{GeV}$. Figure 2 shows the expected and observed exclusion limits as a function of the $\mathrm{H}^{+}$mass hypothesis.

Figure 3 shows $95 \%$ CL exclusion limits set on $\tan \beta$ for the $m_{h}^{\bmod -}$ and the hMSSM scenarios. 


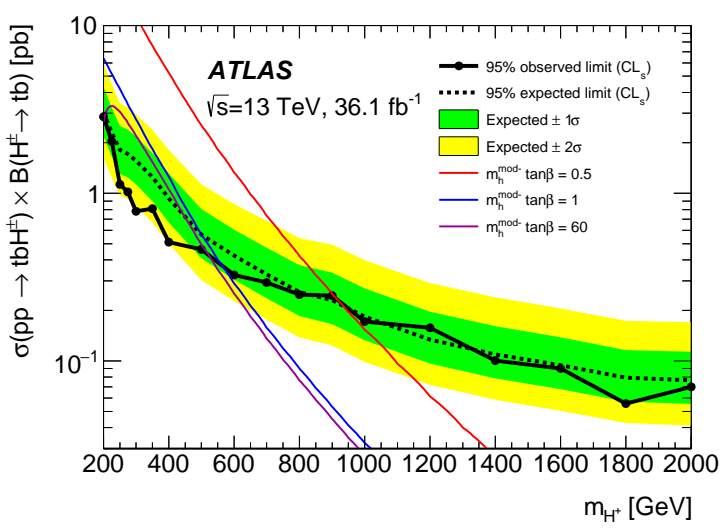

Figure 2: Observed and expected 95\% CL exclusion limits on $\sigma\left(p p \rightarrow t b H^{+}\right) \times \mathscr{B}\left(H^{+} \rightarrow t b\right)$ as a function of the $H^{+}$mass [3].
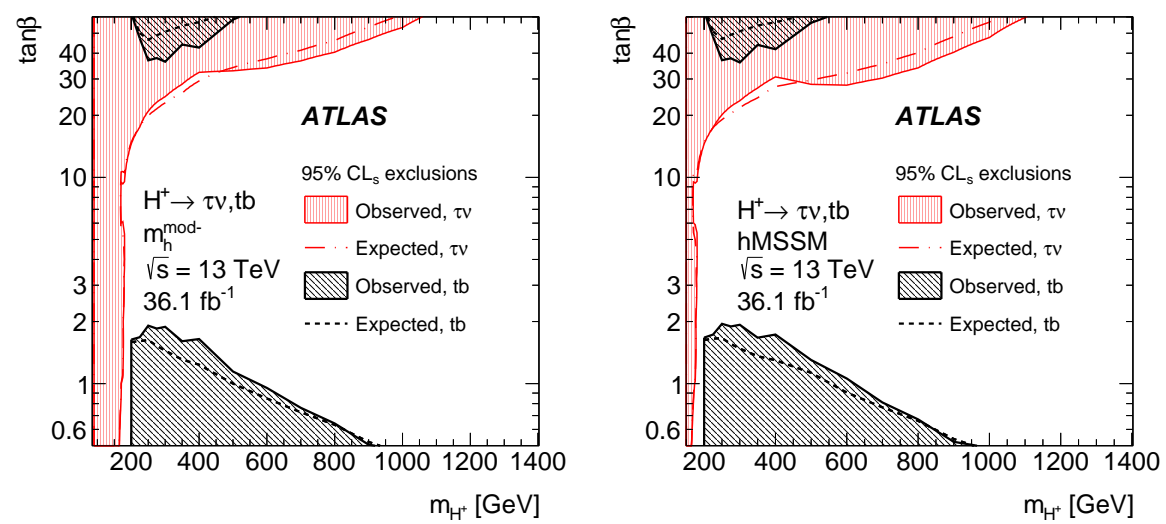

Figure 3: Observed and expected 95\% CL exclusion limits on $\tan \beta$ as a function of $m_{H^{+}}$in the $m_{h}^{\text {mod- }}$ (left) and the hMSSM (right) scenarios. Limits are shown for $\tan \beta$ values in the range $0.5-60$ where predictions are available for both scenarios [3].

\section{References}

[1] ATLAS Collaboration, The ATLAS Detector, JINST 3 (2008) S08003.

[2] ATLAS Collaboration, Search for charged Higgs bosons decaying via $H^{ \pm} \rightarrow \tau^{ \pm} v_{\tau}$ in the $\tau+$ jets and $\tau+$ lepton final states with $36 \mathrm{fb}^{-1}$ of pp collision data recorded at $\sqrt{s}=13 \mathrm{TeV}$ with the ATLAS experiment, JHEP 09 (2018) 139.

[3] ATLAS Collaboration, Search for charged Higgs bosons decaying into top and bottom quarks at $\sqrt{s}$ $=13 \mathrm{TeV}$ with the ATLAS detector, arXiv:1808.03599v1 [hep-ex].

[4] C. Degrande et al., Accurate predictions for charged Higgs production: Closing the $m_{H^{ \pm}} \sim m_{t}$ window, Phys. Lett. B 772 (2017) 87.

[5] A.L. Read, Presentation of search results: the $C L_{s}$ technique, J.Phys. G 28 (2002) 2693. 